\title{
Respiratory Rate Detection Using a Camera as Contactless Sensor
}

\author{
Luca Iozzia $^{1}$, Jesús Lázaro ${ }^{2}$, Eduardo Gil ${ }^{2}$, Luca Cerina $^{1}$, Luca Mainardi ${ }^{1}$, Pablo Laguna ${ }^{2}$ \\ ${ }^{1}$ Dipartimento di Elettronica, Informatica e Bioingegneria, Politecnico of Milan, Milan, Italy \\ ${ }^{2}$ BSICoS Group, Aragón Institute of Enginnering Research (I3A), IIS Aragón, Universidad de \\ Zaragoza, 12, 50018, Zaragoza, Spain
}

\begin{abstract}
Aim of this study is to extract near-continuously respiratory rate by a contactless method. An industrial camera was used to record subjects face. Video data were processed offline to derive the video-photoplethysmographic (videoPPG) signal. Three features were extracted from videoPPG and finger PPG signal: pulse rate variability $(P R V)$, pulse amplitude variability (PAV) and pulse width variability $(P W V)$. A combination of these methods has been exploited to estimate the respiratory rate for each time window of 5 second. The results showed relative error with median around $0.5 \%$ and interquartile range of $5 \%$ both for finger PPG and videoPPG system.
\end{abstract}

\section{Introduction}

The monitoring of breathing rate can be a predictive indicator of adverse events, i.e. cardiac arrest or admission to the intensive care units [1-3]. Different techniques have been explored to monitor respiratory rate. Airflow based methods use attached sensors to the airways like facemask, or mouthpiece to measure the volume of air exhaled. Despite the great accuracy, they result cumbersome and unpractical outside the clinical environment. Thus noninvasive technological alternatives arised as impedance plethysmography and respiratory inductance plethysmography that, by the use of sensors placed on the chest, measure the ribcage movement directly associated to the respiratory process.

However new frontiers such as telemonitoring and stress level monitoring in work environment require minimally invasive technologies that do not need user's active intervention in the measurement.

The video-photoplethysmography (videoPPG) is a technology based on the use of a camera as monitoring device of patient vital parameters. The main benefit is the absence of any sensor attached to the user that makes it suitable for daily monitoring.

Respiratory content modulates the pulse occurrence and the wave morphology in terms of speed and amplitude of
PPG signal. Therefore the extraction of pulse rate variability (PRV), pulse amplitude variability (PAV) and of pulse width variability (PWV) from PPG signal can provide information on the breathing rate. In this work a combination of these methods is presented to recover the respiratory rate.

\section{Materials and Methods}

\subsection{Experimental protocols}

Twenty healthy subjects were recruited for the experiment. They were seated on chair facing the camera device at a distance of approximately $0.5-1 \mathrm{~m}$. An industrial camera was selected with a spatial resolution of $659 \times 494$ pixels and $60 \mathrm{fps}$. The camera was equipped with $15 \mathrm{~mm}$ fixed focal length lens. Simultaneously the PPG signal was recorded from index finger, while the respiratory signal was recorded by a respiratory belt placed on subject's chest. Both signals were sampled at $256 \mathrm{~Hz}$ using the FlexComp Infiniti by Thought Technologies, Inc.

The protocol was the following: 2 minutes of normal breathing (Normal), a period of apnea (whose duration depended on subject's capacity), 2 minutes of recovery (Recovery) and a phase of controlled breath at 10 breaths per minute (Controlled).

\subsection{VideoPPG signal extraction}

Videos were saved in RGB, uncompressed, AVI raw format and processed offline to derive the videoPPG signal according to the algorithm presented in [4]. Three regions of interests (ROI) were considered: forehead, nose and cheek. ROI detection and tracking were performed respectively by the Viola-Jones face detection algorithm [5] while ROI tracking along X-Y axis by the LKT motion flow tracking algorithm [6].

Within each ROI a spatial average of pixel intensities of each channel (red, green and blue) has been calculated for each frame to generate $N$ raw signals (where $N$ is the num- 


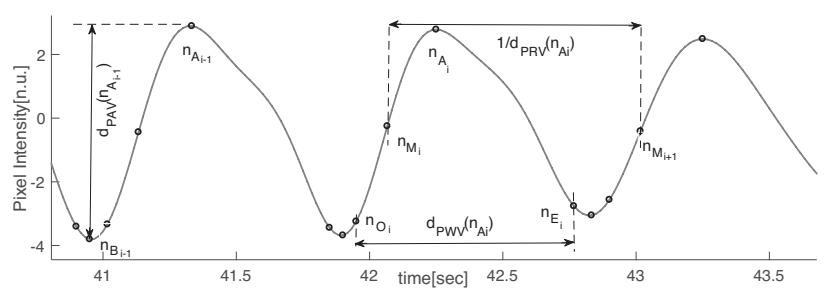

Figure 1. Derived respiratory signals defined by a set of fiducial points.

ber of color channels). Considering $M$ as the number of ROI, a total of $M * N$ raw traces is obtained. The time series were first detrended [7] and band-pass filtered with a cut-off frequencies of $f_{1}=0.1 \mathrm{~Hz}$ and $f_{2}=5 \mathrm{~Hz}$. To enhance the pulsatile component of the reflected light and reduce the motion noise, the ZCA method [8] was applied in each ROI. As a result, $M$ videoPPG signals were obtained. Among them, the selection of the target videoPPG signal was achieved by calculating the power spectral density (PSD) on the entire signal and by measuring the SNR using the following formula:

$$
S N R=10 * \log _{10}\left(\frac{\int_{f_{1}}^{f_{2}} \operatorname{PSD}(\mathrm{f}) \mathrm{df}}{\int_{0.1}^{f_{1}} \operatorname{PSD}(\mathrm{f}) \mathrm{df}+\int_{\mathrm{f}_{2}}^{4} \operatorname{PSD}(\mathrm{f}) \mathrm{df}}\right)
$$

where $P S D(f)$ with $i \in\left\{1,2, \ldots, N_{R O I}\right\}$ is the PSD of $i^{t h}$ videoPPG signals, $f_{1}=f_{c}-0.15 \mathrm{~Hz}, f_{2}=f_{c}+$ $0.15 \mathrm{~Hz}$ and where $f_{c}$ is the cardiac frequency (measured in $\mathrm{Hz}$ ). The signal with the highest SNR was selected as target videoPPG signal.

\subsection{Detection of fiducial points}
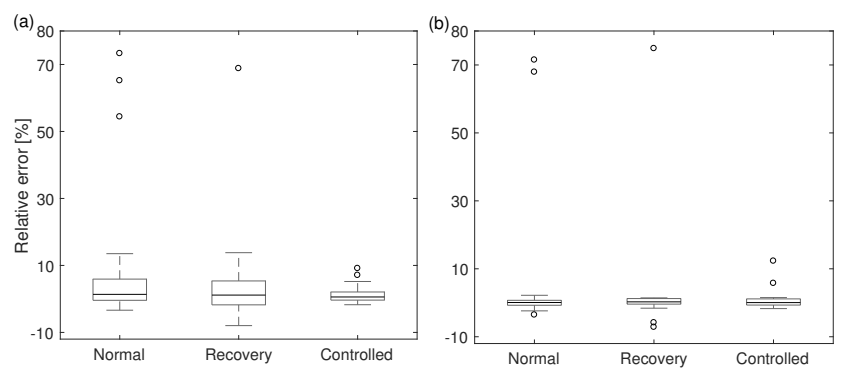

Figure 2. Boxplot of the respiratory rate relative error obtained respectively with videoPPG (a) and fPPG (b) system.

The method implemented by [9] was applied to derive Pulse Width Variability (PWV), Pulse Rate Variability (PRV) and Pulse Ampitude Variability (PAV) both from
fingerPPG (fPPG) and videoPPG signals. Briefly the systolic peaks $n_{A_{i}}$ were identified by an automatic PPG pulse detector based on a low-pass filter differentiator and an adaptive time-varying threshold [10]. Next the dyastolic points $n_{B_{i}}$ were identified inside a temporal window previous to $n_{A_{i}}$ :

$$
n_{B_{i}}=\underset{n}{\operatorname{argmin}}\{s(n)\}, n \in\left[n_{A_{i}}-0.3 f_{s}, n_{A_{i}}\right] \text {, }
$$

where $s(n)$ is the PPG signal and $f_{s}$ the sampling frequency of the signal. Finally $n_{M_{i}}$ was detected as the half of the rising edge of the cardiac pulse:

$n_{M_{i}}=\underset{n}{\operatorname{argmin}}\left\{s(n)-\frac{s\left(n_{A_{i}}\right)+s\left(n_{B_{i}}\right)}{2}\right\}, n \in\left[n_{B_{i}}, n_{A_{i}}\right]$,

The width of each pulse wave was measured by considering the onset $n_{O i}$ and the end of the wave $n_{E_{i}}$, while the distances between $n_{M_{i+1}}$ and $n_{M_{i}}$ are used to estimate the pulse rate (see Fig. 1). More details are shown in [11].

\subsection{Derived respiration signals}

Three derived respiration (DR) signals were calculated using pulse-to-pulse methods PRV, PAV and PWV. The DR signal based on PRV was obtained as pulse-to-pulse series:

$$
d_{P R V}^{u}(n)=\sum_{i} f_{s} \frac{1}{n_{N_{i}}-n_{N_{i-1}}} \delta\left(n-n_{A_{i}}\right)
$$

where ' $u$ ' indicates that the signal was unevenly sampled, and $n_{N_{i}}$ represents the arrival of pulse after removing the ectopic and missdetected pulses from $n_{M_{i}}$ by using the method proposed by Mateo et al [12]. The other two derived signals based on PAV and PWV were obtained as follows:

$$
\begin{gathered}
d_{P A V}^{u}(n)=\sum_{i}\left[s\left(n_{A_{i}}\right)-s\left(n_{B_{i}}\right)\right] \delta\left(n-n_{A_{i}}\right) \\
d_{P W V}^{u}(n)=\sum_{i} \frac{1}{f_{s}}\left[n_{E_{i}}-n_{O_{i}}\right] \delta\left(n-n_{A_{i}}\right)
\end{gathered}
$$

An outlier rejection rule based on median absolute deviation was applied [13] and the signals were sampled at $4 \mathrm{~Hz}$ using the cubic spline interpolation. A band-pass filter was applied with the cutoff frequencies $f_{1}=0.075$ and $f_{2}=1 H z$.

\subsection{Respiratory rate estimation}

The respiratory rate has been estimated from DR signals every $5 s$ using a running window of $30 s$ length by adapting the method described in [9]. 

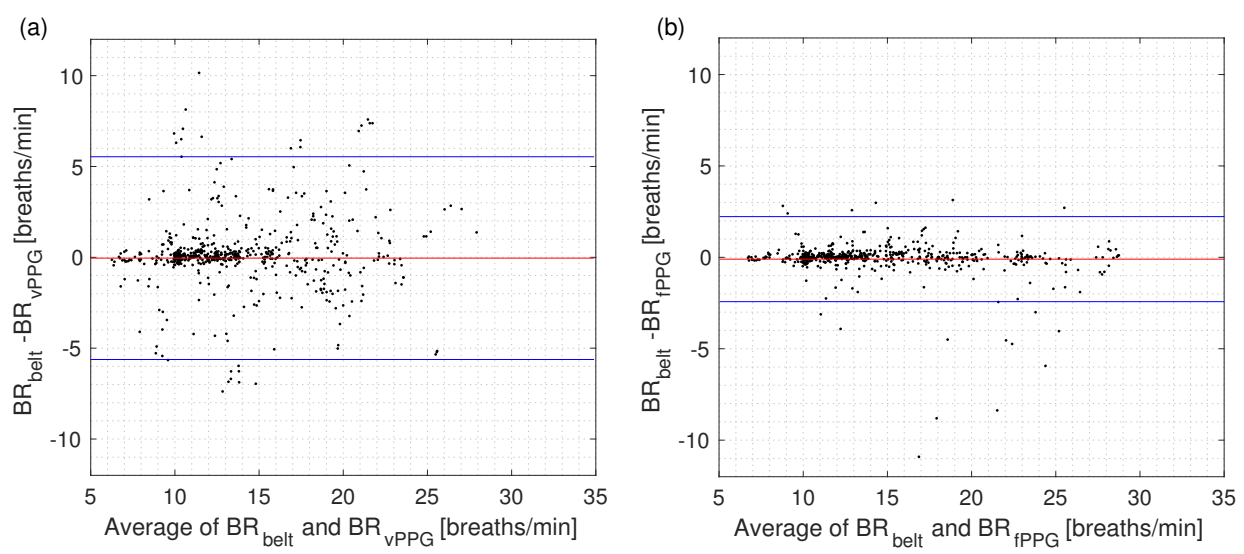

Figure 3. Bland-Altman plot illustrating the agreement between instantaneous breath rate measured by the respiratory belt reference and vdieoPPG system (a) and by the respiratory belt reference and fPPG (b).

Briefly the method uses a combination of PWV, PAV, PRV. Let's indicate $S_{j}$ the power spectral densities for each DR signal. From this spectra an average is computed as:

$$
\bar{S}(f)=\sum_{i} \chi_{j}^{A} \chi_{j}^{B} S_{j}(f)
$$

where $\chi^{A}$ and $\chi^{B}$ are two weighting factors used to include in the average $\bar{S}(f)$ only peaked spectra. For this reason the following quantity $P_{j}$ has been included:

$$
P_{j}=\frac{\int_{f_{p}(j)-0.05 H z}^{f_{p}(j)+0.05 \mathrm{~Hz}} S_{j}(f) \mathrm{df}}{\int_{0.15 \mathrm{~Hz}}^{0.5 \mathrm{~Hz}} S_{j}(f) \mathrm{df}}
$$

where $P_{j}$ is the percentage of power of $S_{j}$ contained in the interval centered around the highest peak $\left(f_{p}(j)\right)$ respected to the total power contained in $[0.15-0.5] \mathrm{Hz}$. Peaked spectra are defined as those which have $P_{j}$ greater than a threshold defined by $\xi=40 \%$, thus:

$$
\chi_{j}^{A}=\left\{\begin{array}{l}
1, P_{j} \geq \xi \\
0, \text { otherwise }
\end{array}\right.
$$

while the condition $\chi^{B}$ selects the $S_{j}$ whose $P_{j}$ is not less than $\lambda=30 \%$ of the maximum $P_{j}$ :

$$
\chi_{j}^{B}=\left\{\begin{array}{l}
1, P_{j} \geq \max \left\{P_{j}\right\}-\lambda \\
0, \text { otherwise }
\end{array}\right.
$$

The respiratory frequency $\hat{f}$ is finally calculated as the frequency that corresponds to the maximum of $\bar{S}(f)$ in the frequency band $[0.15-0.5] \mathrm{Hz}$ :

$$
\hat{f}=\underset{f \in[0.15-0.5]}{\operatorname{argmax}}\{\bar{S}(f)\}
$$

After initialization, the frequency band for the searching of $\hat{f}(i)$ was centered around the previous estimated value $\hat{f}(i-1)$. If no spectra is enough peaked, the search of $\hat{f}$ is performed in a larger frequency band $[0.09-0.55] \mathrm{Hz}$.

The overall results have been expressed according to the relative error $(\%)$ :

$$
\epsilon_{R}=\frac{\hat{f}-f_{R}}{f_{R}} \times 100
$$

where $\hat{f}$ is the estimated respiratory frequency and $f_{R}$ is the respiratory frequency obtained from the respiratory belt used as a reference for the analysis of PPPG and videoPPG.

Moreover it has been quantified the respiratory modulation strength contained in derived breathing signal for each method (PAV, PRV and PWV). The following measure has been obtained considering the percentage of spectra that contributed in the calculation of the averaged spectra.

\section{Results}

The boxplot of relative errors have been expressed for videoPPG system (Fig.2(a)) and for the fPPG system (Fig.2(b)) according to the three different conditions Normal, Recovery and Controlled. The fPPG system outperformed the videoPPG one in all the conditions: the median/interquartile (IQR) range in $\mathrm{PPG}$ are maintained below the $2 \%$, while in the videoPPG the median/IQR is higher (in Normal 1.36/6.33 \%, in Recovery 1.14/7.14 \% and in Controlled breath 0.58/2.43\%). Both systems had the worst performance $(\epsilon>15 \%)$ for the same subjects (subject 1 and 15). Interestingly the following subjects showed a respiratory frequency higher than $0.35 \mathrm{~Hz}$ in Normal and Recovery phase.

Bland-Altman plots in Fig.3 show the accuracy of instantaneous breathing rate (BR) measurements (calculated every $5 s$ and expressed as breath/min) of the whole population comparing the videoPPG method with the reference 
Table 1. Percentage of spectra taking part to the estimation of breathing rate for videoPPG system.

\begin{tabular}{lccc}
\hline \hline Condition & PAV & PRV & PWV \\
\hline Normal & 45.96 & 46.66 & 40.46 \\
Recovery & 40.47 & 51.11 & 38.13 \\
Controlled & 83.86 & 35.43 & 51.23 \\
\hline \hline
\end{tabular}

respiratory belt (Fig.3(a)) and the fPPG method with the gold standard (Fig.3(b)). In each Bland-Altman plot the centered line represents the bias and the other two lines represent the $95 \%$ limits of agreement. In videoPPG system the bias is equal to -0.04 breaths $/$ min , with $95 \%$ of agreement inside \pm 5.53 breaths $/ \mathrm{min}$. Considering the fPPG system the bias is -0.10 breaths $/ \mathrm{min}$, while the $95 \%$ of instantaneous breathing rate values are contained in the range \pm 2.22 breaths/min.

\section{Discussions and Conclusions}

We proposed a fully automatic method based on contactless camera-based technology to extract instantaneous breathing information and compared the results to the ones obtained from fPPG system.

Considering Tabs. 1 and 2, in the videoPPG the respiratory content was almost equally distributed between all the methods for Normal and Recovery condition. In Controlled breath, the respiratory content was more expressed in the breathing signal derived from PAV method. While for the fPPG system, the PWV method enhanced the respiratory modulation more than the other two methods during Normal and Recovery session, while in Controlled breath the respiratory content was mainly included in PRV method.

The overall results look promising, despite the respiratory modulation looks to be less evident for higher RR values. Future work is needed to investigate this aspect more sistematically on a database which includes controlled breathing frequencies at different values.

\section{References}

[1] Subbe CP, Davies RG, Williams E, Rutherford P, Gemmell L. Effect of introducing the modified early warning score on clinical outcomes, cardio-pulmonary arrests and intensive care utilisation in acute medical admissions. Anaesthesia 2003;58(8).

[2] Fieselmann JF, Hendryx MS, Helms CM, Wakefield DS. Respiratory rate predicts cardiopulmonary arrest for internal medicine inpatients. J Gen Intern Med 1993;8(7):354360 .

[3] McBride J, Knight D, Piper J, Smith GB. Long-term effect
Table 2. Percentage of spectra taking part to the estimation of breathing rate for fPPG system.

\begin{tabular}{lccc}
\hline \hline Condition & PAV & PRV & PWV \\
\hline Normal & 46.66 & 54.73 & 65.50 \\
Recovery & 44.91 & 51.34 & 70.64 \\
Controlled & 28.07 & 86.31 & 19.29 \\
\hline \hline
\end{tabular}

of introducing an early warning score on respiratory rate charting on general wards. Resuscitation 2005;65(1):41 44.

[4] Iozzia L, Cerina L, Mainardi L. Relationships between heart-rate variability and pulse-rate variability obtained from video-ppg signal using zca. Physiol Meas 2016; 37:1934-1944.

[5] Viola P, Jones M. Rapid object detection using a boosted cascade of simple features. Proceedings of IEEE Conference on Computer Vision and Pattern Recognition 2001; 511-517.

[6] Lucas BD, Kanade T. Detection and tracking of point features. Technical Report MU CS 91132 Carnegie Mellon University 1991;

[7] Tarvainen MP, Ranta-Aho PO, Karjalainen PA. An advanced detrending method with application to HRV analysis. IEEE Trans Biomed Eng 2002;49:172-175.

[8] Bell AJ, Sejnowski TJ. The 'Independent Components' of natural scenes are edge filters. Vision Res 1997;37:33273338.

[9] Lázaro J, Nam Y, Gil E, Laguna P, Chon KH. Respiratory rate derived from smartphone-camera-acquired pulse photoplethysmographic signals. Physiol Meas 2015; 36(11):2317-2333.

[10] Lázaro J, Gil E, Vergara JM, Laguna P. Pulse rate variability analysis for discrimination of sleep-apnea-related decreases in the amplitude fluctuations of pulse photoplethysmographic signal in children. IEEE J Biomed Health Inform 2014;18:240-246.

[11] Lázaro J, ALcaine A, Romero D, Gil E, Laguna P, Pueyo E, Bail'on R. Electrocardiogram derived respiratory rate from QRS slopes and R-wave angle. Ann Biomed Eng 2014; 42:2072-2083.

[12] Mateo J, Laguna P. Analysis of heart rate variability in the presence of ectopic beats using heart timing signal. IEEE Trans Biomed Eng 2003;50:334-343.

[13] Bailón R, Sörnmo L, Laguna P. A robust method for ecgbased estimation of the respiratory frequency during stress testing. IEEE Trans Biomed Eng 2006;53:1273-1285.

Address for correspondence:

Luca Iozzia

luca.iozzia@polimi.it

Via Golgi 39, Milan, Italy 